\title{
A Quantitatively Derived NMAC Analog for Smaller Unmanned Aircraft Systems Based on Unmitigated Collision Risk
}

Andrew Weinert

\author{
RTCA SC-147
}

March 12, 2020

LINCOLN LABORATORY

Sponsored by FAA AUS-300 UAS Integration Office 


\section{Legal Notices}

DISTRIBUTION STATEMENT A. Approved for public release. Distribution is unlimited.

This material is based upon work supported by the Federal Aviation Administration under Air Force Contract No. FA8702-15-D-0001. Any opinions, findings, conclusions or recommendations expressed in this material are those of the author(s) and do not necessarily reflect the views of the Federal Aviation Administration.

This document is derived from work done for the FAA (and possibly others), it is not the direct product of work done for the FAA. The information provided herein may include content supplied by third parties. Although the data and information contained herein has been produced or processed from sources believed to be reliable, the Federal

Aviation Administration makes no warranty, expressed or implied, regarding the accuracy, adequacy, completeness, legality, reliability or usefulness of any information, conclusions or recommendations provided herein. Distribution of the information contained herein does not constitute an endorsement or warranty of the data or information provided herein by the Federal Aviation Administration or the U.S. Department of Transportation. Neither the Federal Aviation Administration nor the U.S. Department of Transportation shall be held liable for any improper or incorrect use of the information contained herein and assumes no responsibility for anyone's use of the information. The Federal Aviation Administration and U.S. Department of Transportation shall not be liable for any claim for any loss, harm, or other damages arising from access to or use of data or information, including without limitation any direct, indirect,

incidental, exemplary, special or consequential damages, even if advised of the possibility of such damages. The Federal Aviation Administration shall not be liable to anyone for any decision made or action taken, or not taken, in reliance on the information contained herein

(C) 2020 Massachusetts Institute of Technology

Delivered to the U.S. Government with Unlimited Rights, as defined in DFARS Part 252.227-7013 or 7014 (Feb 2014). Notwithstanding any copyright notice, U.S. Government rights in this work are defined by DFARS 252.227-7013 or

DFARS 252.227-7014 as detailed above. Use of this work other than as specifically authorized by the U.S.

Government may violate any copyrights that exist in this work. 


\section{Metric to Evaluate Collision Avoidance Systems}

- Aircraft must operate as to not create a MAC hazard due to the loss of life and property

- Collision avoidance systems are mandated to minimize the MAC risk between aircraft

- Systems defined by sets of performance requirements

- Separation criteria dependent upon the types of encountering aircraft

- Fast-time modeling and simulation routinely used to evaluate these systems

- MAC statistics are difficult to estimate, so NMACs are used as a preferred metric

- Defined as a loss of separation $\mathbf{5 0 0}$ feet horizontally and 100 feet vertically

- Acts as a "measuring stick" for assessing the goodness of a system

- Originally quantitatively defined to support safety evaluations of TCAS in the 1980s

- NMAC was defined using assumptions for encounters with only manned aircraft, these assumptions are not representative of UAS-only encounters

- NMAC is a potential but not a reasonable metric to evaluate smaller UAS safety 


\section{Objective}

Objective: Propose a sNMAC criteria that can be used to evaluate safety systems that mitigate the likelihood of a MAC for encounters between smaller UASs

- Quantitatively define what constitutes a close encounter between smaller UASs in simulation

- Support safety modeling and simulation efforts, specifically RTCA SC-147

- Exclude incident reporting criteria from initial scope

- Functionally analogous to NMAC's role in evaluating safety systems for manned aircraft

- Preliminary SNMAC identified at SME forum attended

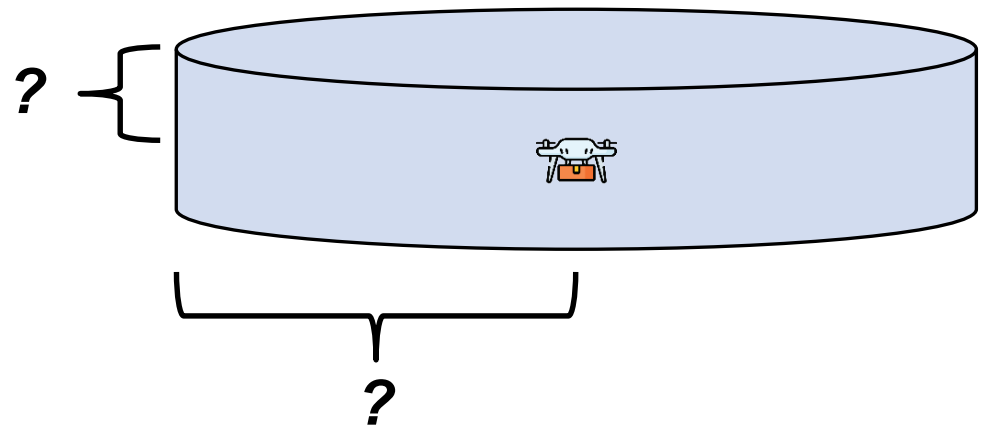

Key contribution will be quantitative definitions for horizontal and vertical separation only by JHU APL, MIT LL, and MITRE 


\section{sNMAC for UAS-only Encounters}

Encounters Different that for Manned Aircraft

- The size of a given UAS is generally significantly smaller than a manned aircraft, with many low altitude smaller UASs having a wingspan of 15 feet or less

- The altitudes, closing speeds, and dynamics of UAS-only encounters are significantly different than encounters with manned aircraft

- Expected to occur at lower altitudes of 1200-500 feet AGL or less

- Different aircraft flight performance and dynamics

Smaller UAS vs Large UAS / Manned Aircraft

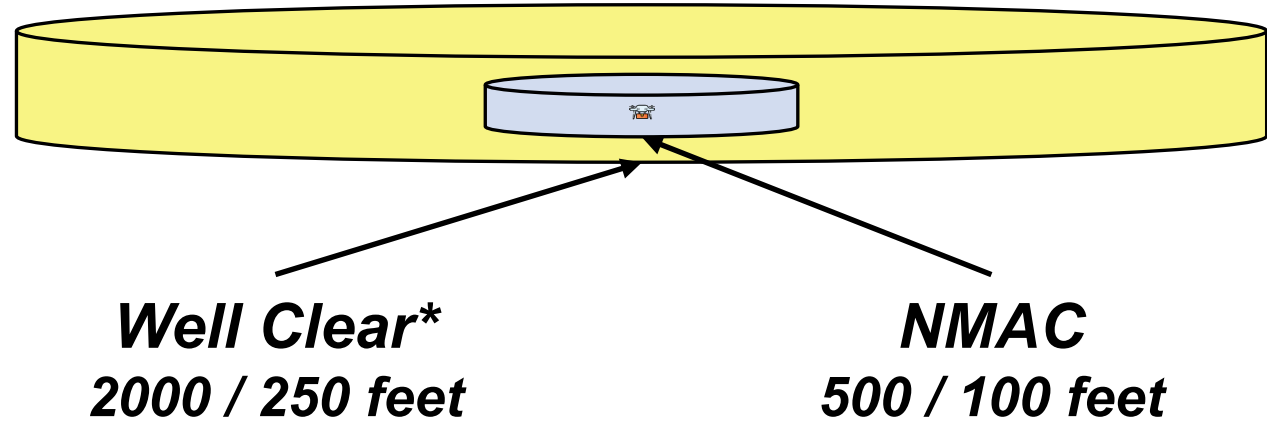

Smaller UAS vs Smaller UAS

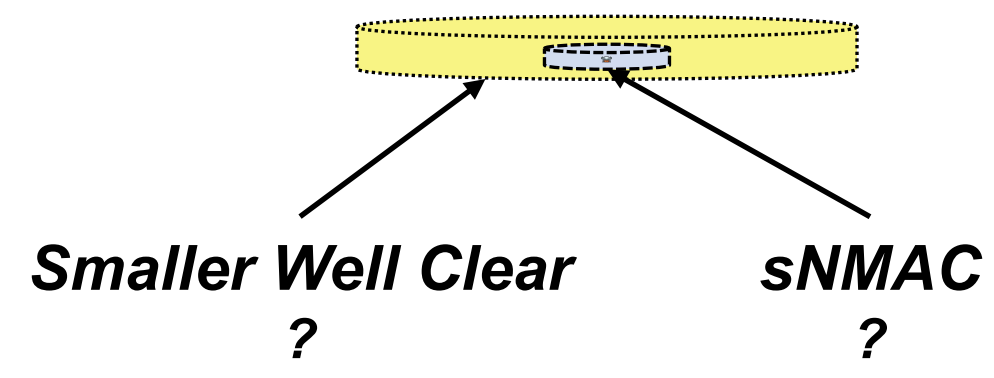


- Overview

- NMAC History

- sNMAC Assumptions

- Method and Results

- Conclusion 
"One of the first tasks [is] to specify the limits within which aircraft may approach each other during flight without being considered as a near misses."

"It will also be necessary to provide a standard basis for estimating when these limits have been breached. Whether we have 150, 1500, or 15,000 near misses will, in large measure, depend on our standards."

"When standards are established and a stable "near miss" history develops, it will be possible to systematically explore the effect if various methods designed to reduce exposure to collision"

-Dr. C.P. Seitz, USNAVY ONR 


\section{System Safety Study of TCAS II NMAC Quantitatively Defined}

\section{(11)}

II

"[NMAC is defined as] at the closet point of approach, the vertical separation is less than 100 feet and the horizontal separation is less than 500 feet."

Physical dimensions of the aircraft intended to be equipped with TCAS was a main consideration when defining NMAC

NMAC criteria largely adopted by aviation community and TCAS successor also assessed using NMAC 


\section{NMAC Assumptions and Intrinsic Qualities}

- An NMAC is considered a critical severity incident

- Should be rare and mitigated against

- Used as a MAC surrogate because it is difficult to calculate MAC statistics directly

- The unmitigated likelihood of a MAC given an NMAC, P(MAC|NMAC), should be low

- Historically assumed to be $10 \%$

- Kochenderfer et al. demonstrated likelihood dependent upon aircraft size and could be as low as $2 \%$

- NMAC is a static volume, it does not change depending upon performance or operations

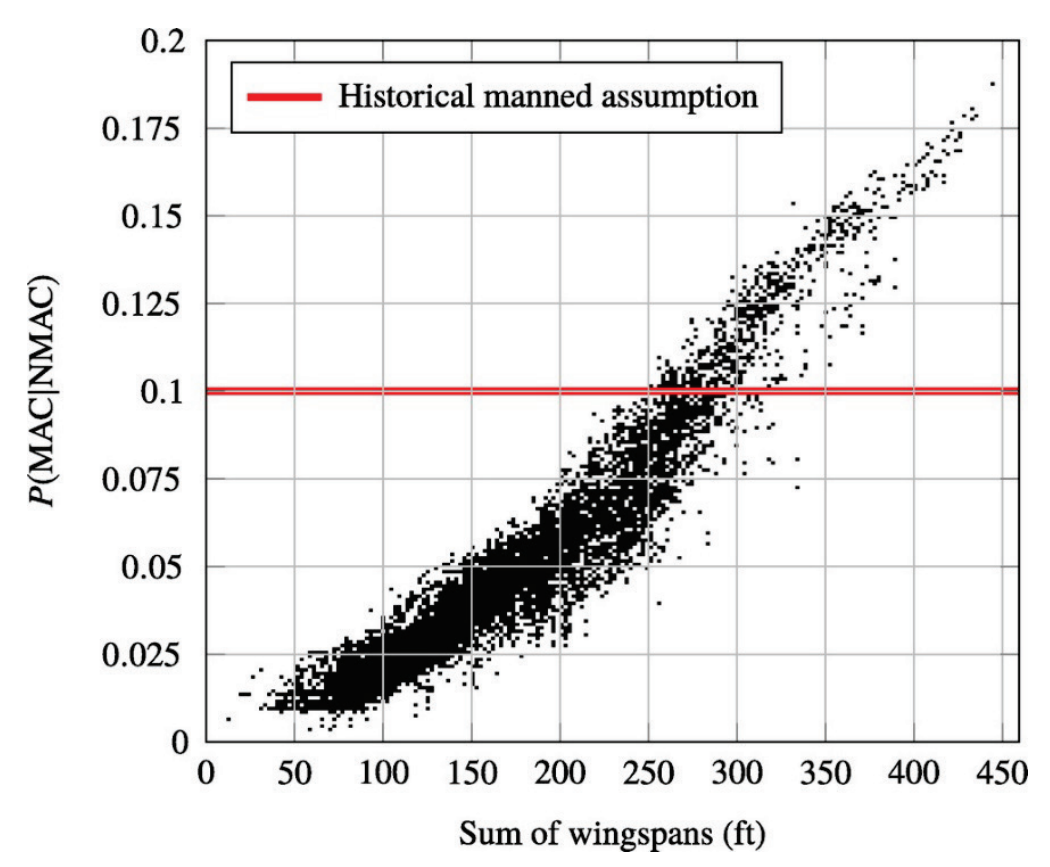

Unmitigated P(MAC|NMAC) as a function of the sum of wingspans of manned aircraft equipped without TCAS* 
- Overview

- NMAC History

- sNMAC Assumptions

- Method and Results

- Conclusion 


\section{sNMAC Form Assumptions - Preliminary}

- Applicable to all smaller UAS based on wingspan

- No consideration for or enforcing a 5:1 horizontal to vertical ratio like NMAC

- No technical justification for a 5:1 ratio based on NMAC literature review

- Uniform and consistent across all use cases

- Should not vary based on altitude, aircraft performance, location, operating limitations, time, or wake turbulence, etc.

- No consideration for small UAS operating limits defined by 14 CFR $\S 107.51$

- Aligned with aviation norms, components should be a multiple of 5, such as 25 feet

- Position error initially not considered; final criteria will include more considerations

- No upfront consideration for the 20 feet altitude accuracy in the Remote ID NPRM*

- Violation reporting and flight testing implications could also be considered

- Quantization only considered for rounding purposes 


\section{Smaller UAS}

\section{Defining Aircraft Based on Wingspan, not Weight}

- "Small UAS" are defined as UAS platforms weighing $\leq 55$ pounds MGTOW

- Aligns with 14 CFR §107.3

- Originally based on decades old military classifications for UAS

- "Smaller UAS" are defined as an UAS with a wingspan $\leq 25$ feet

- Aligns with draft ASTM F38 WK62668 and RTCA SC-147 DAA performance standards

- Includes all currently advertised active small UAS with MGTOW $\leq 55$ pounds

- Wingspan limit based on maintaining assumptions of recommendations for separation assurance criteria between UAS and manned aircraft ${ }^{*}$

- Definition not based on transponder or equipage requirements

- sNMAC applicability limited to smaller UAS

- The likelihood of a MAC is dependent upon aircraft size

- Maximum smaller UAS height assumed to be 12 feet, based on AUVSI robotics database

- No recommended altitude and MGTOW constraints for SNMAC 


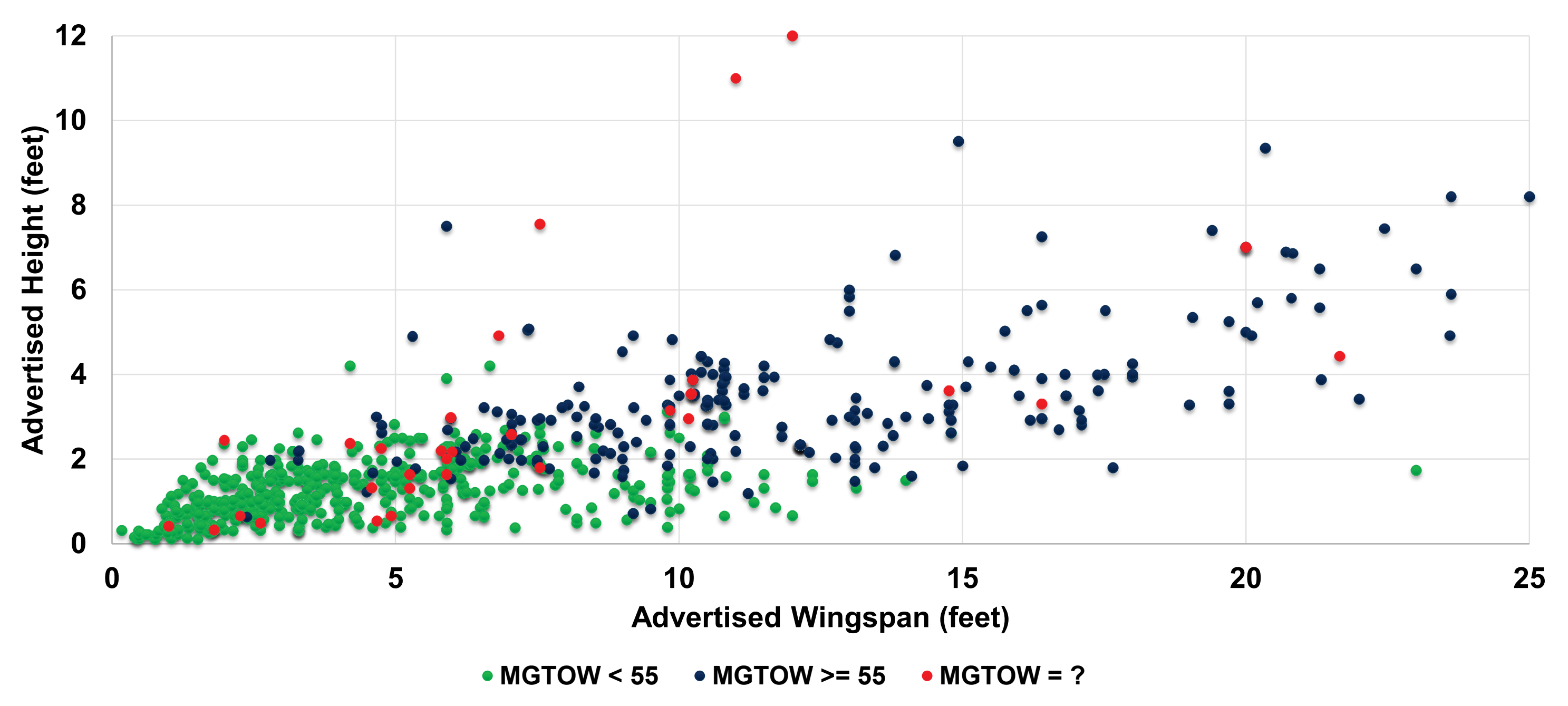




\section{sNMAC Minimum Dimensions Based on Assumed Maximum Effective Width and Height}

Maximum Width (wingspan)

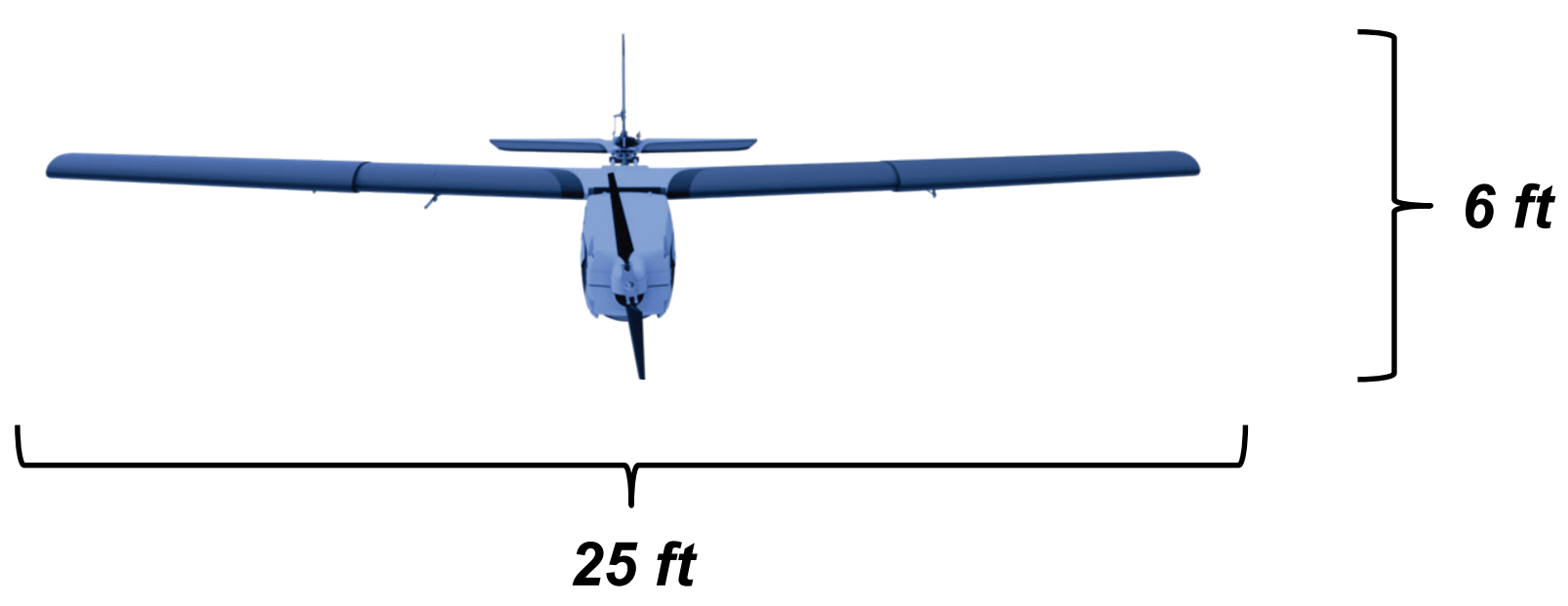

Horizontal separation should be at least $50 \mathrm{ft}(25+25)$ and represent the sum of wingspans

Vertical separation should be at least $15 \mathrm{ft}$ based on rounding effective height when banking or sum of most of heights
Maximum Effective Height

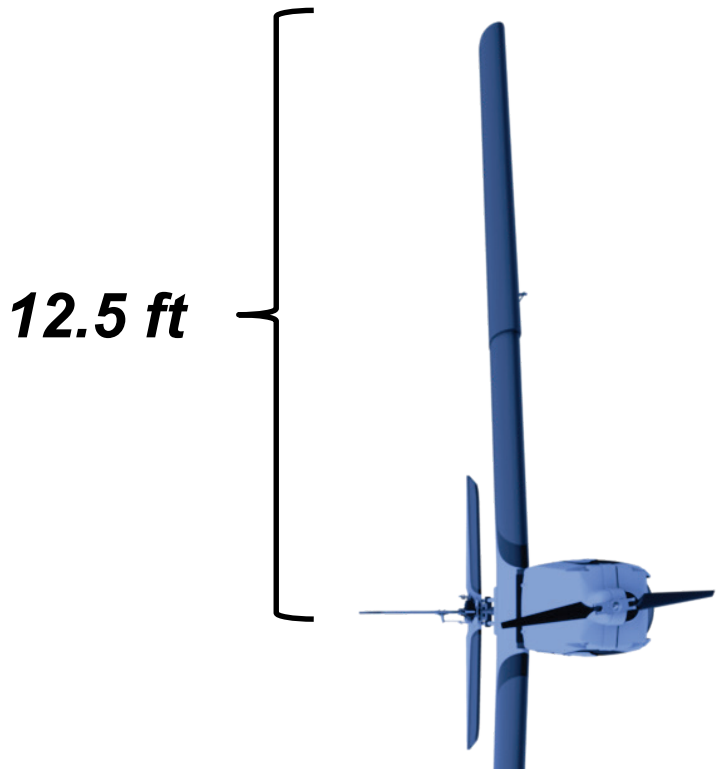


- Overview

- NMAC History

- sNMAC Assumptions

- Method and Results

- Conclusion 


\section{Method}

1. Select independent minimum and maximum limits for wingspan and height

2. Select independent distribution types for wingspan and height

3. Generate a set of horizontal and vertical miss distances

4. Drawing from wingspan and height distributions (\#2) assess in a first-order physics simulation the likelihood of MAC given horizontal and vertical miss distances (\#3)

5. Visualize likelihood of a MAC given \#4

6. Downselect sNMAC candidates that support $P(M A C \mid s N M A C) \leq 0.1$

7. Identify preliminary SNMAC at SME forum 


\section{Horizontal Miss Distance Distributions Consistent Uniform Height Distribution}

Uniform

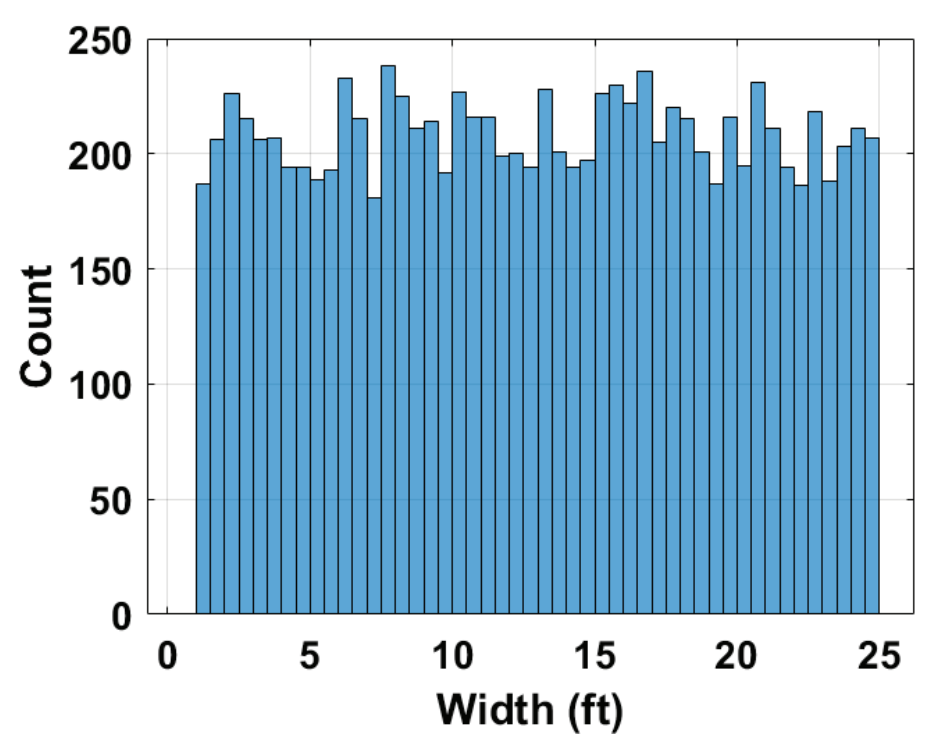

Left Skewed

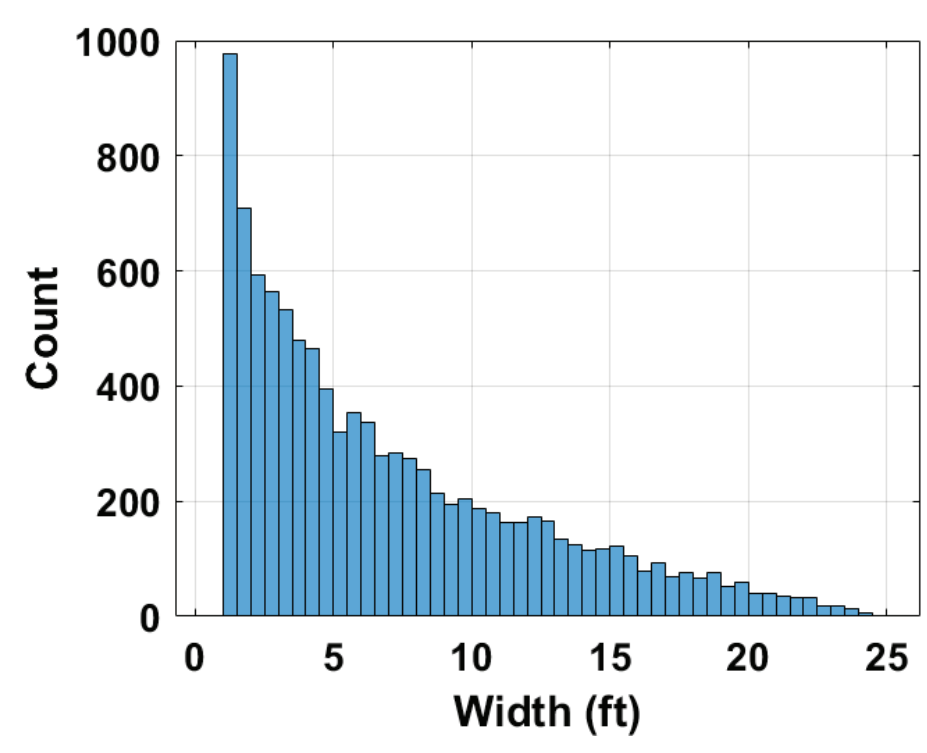

Right Skewed

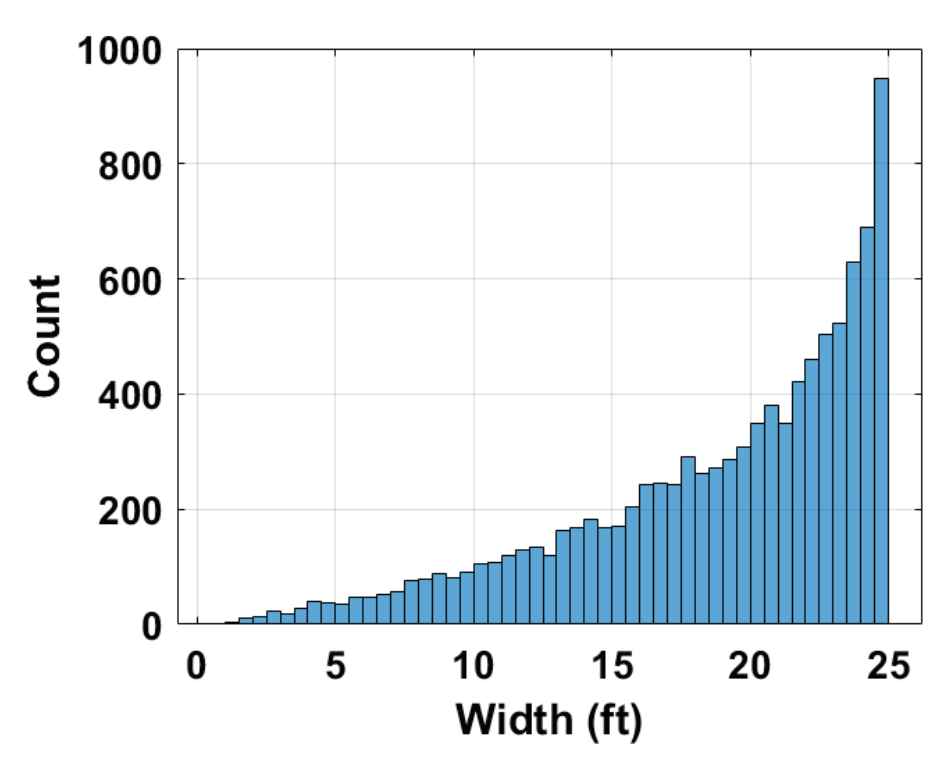

Varying the width distributions enables assessing the sensitivity of P(MAC | SNMAC) given different potential smaller UAS flight compositions 


\section{How to Interpret P(MAC | sNMAC) Results}

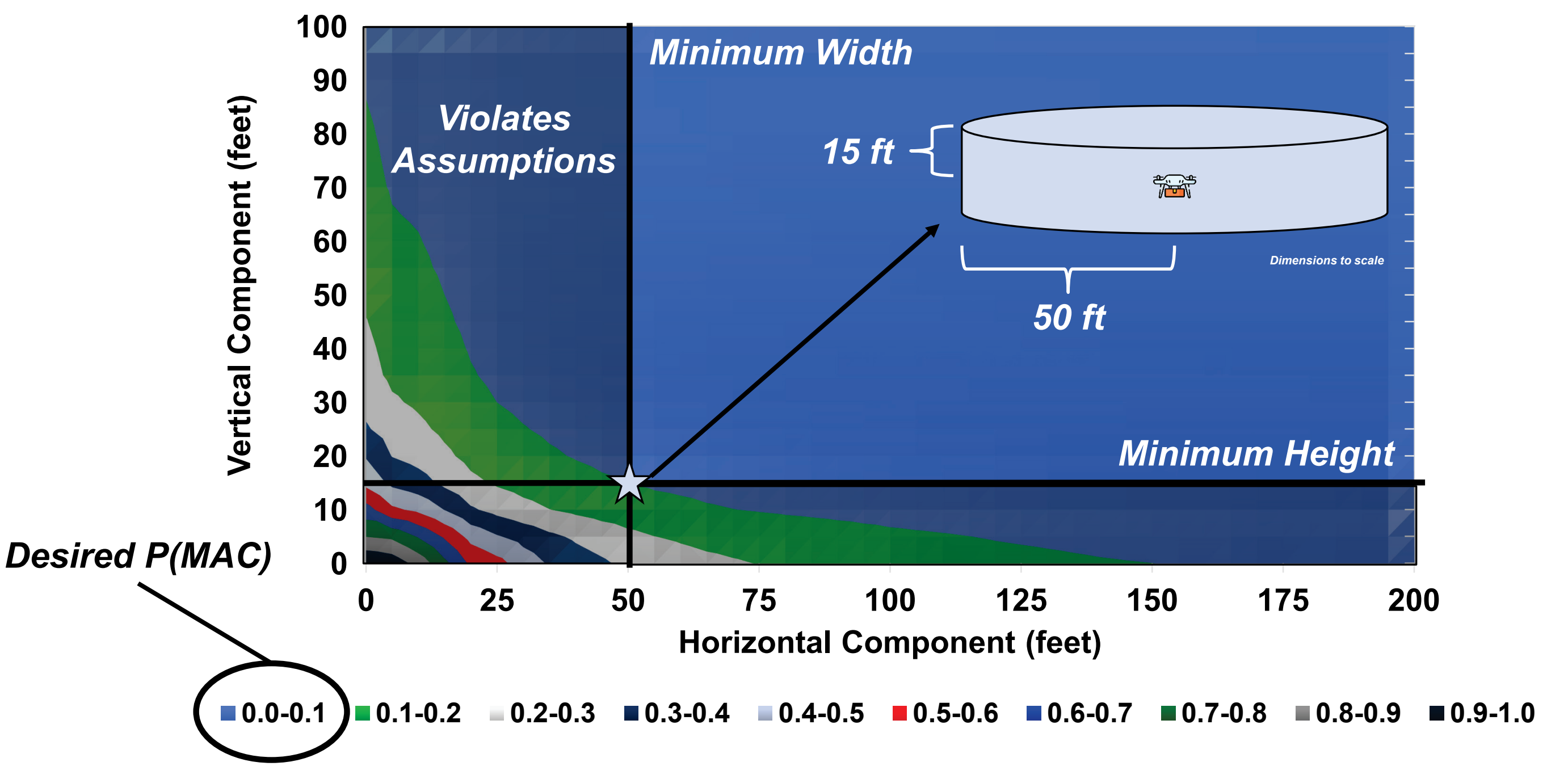




\section{Width - Uniform, Height - Uniform}

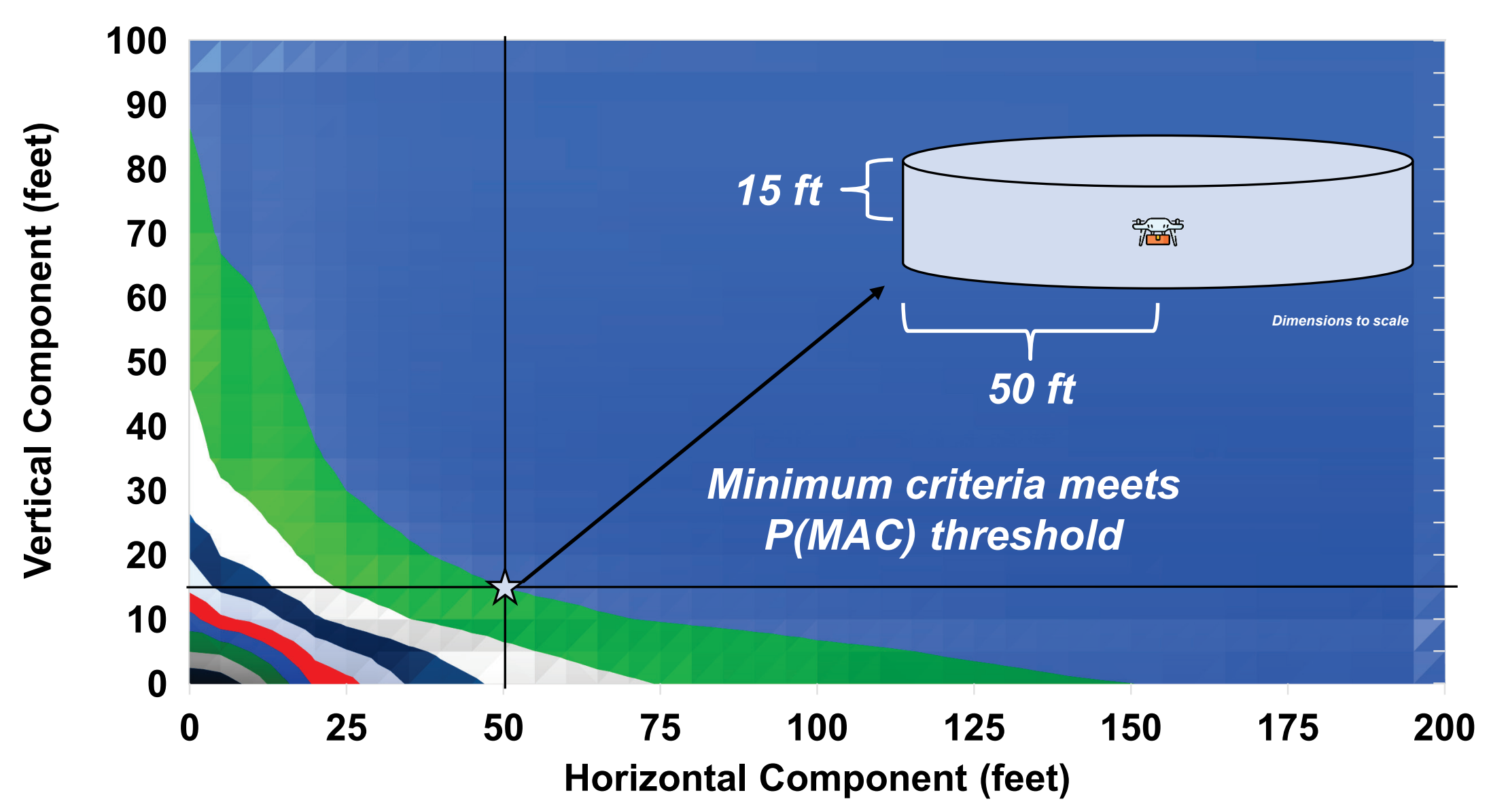

$\varpi 0.0-0.1 \square 0.1-0.2 \square 0.2-0.3 \square 0.3-0.4 \square 0.4-0.5 \square 0.5-0.6 \square 0.6-0.7 \square 0.7-0.8 \backsim 0.8-0.9 \square 0.9-1.0$ 


\section{P(MAC | sNMAC)}

\section{Width - Left Skewed, Height - Uniform}

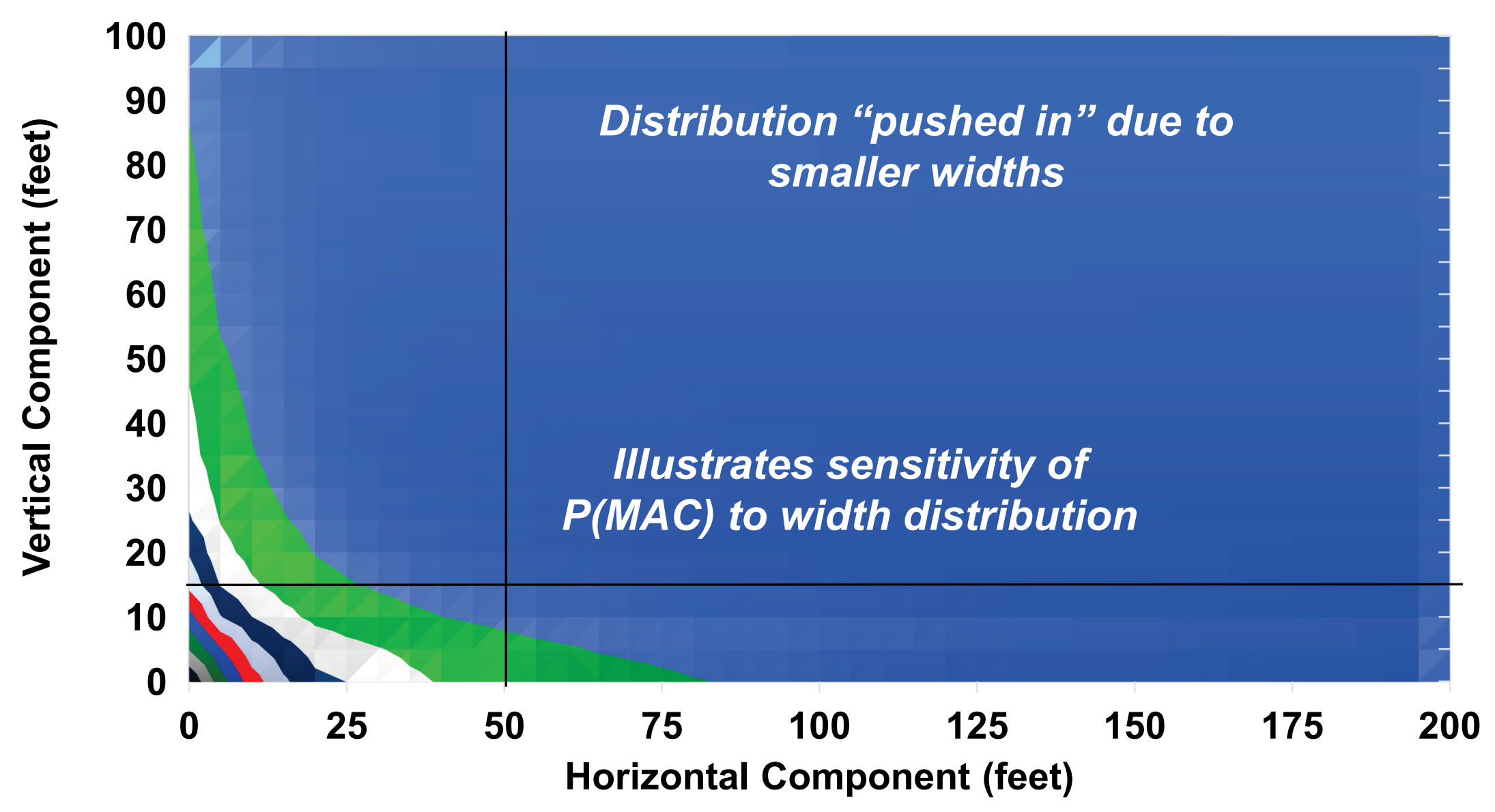

$\backsim 0-0.1 \backsim 0.1-0.2 \quad 0.2-0.3 \square 0.3-0.4 \square 0.4-0.5 \square 0.5-0.6 \square 0.6-0.7 \backsim 0.7-0.8 \backsim 0.8-0.9 \square 0.9-1$ 


\section{P(MAC | SNMAC)}

\section{Width - Right Skewed, Height - Uniform}

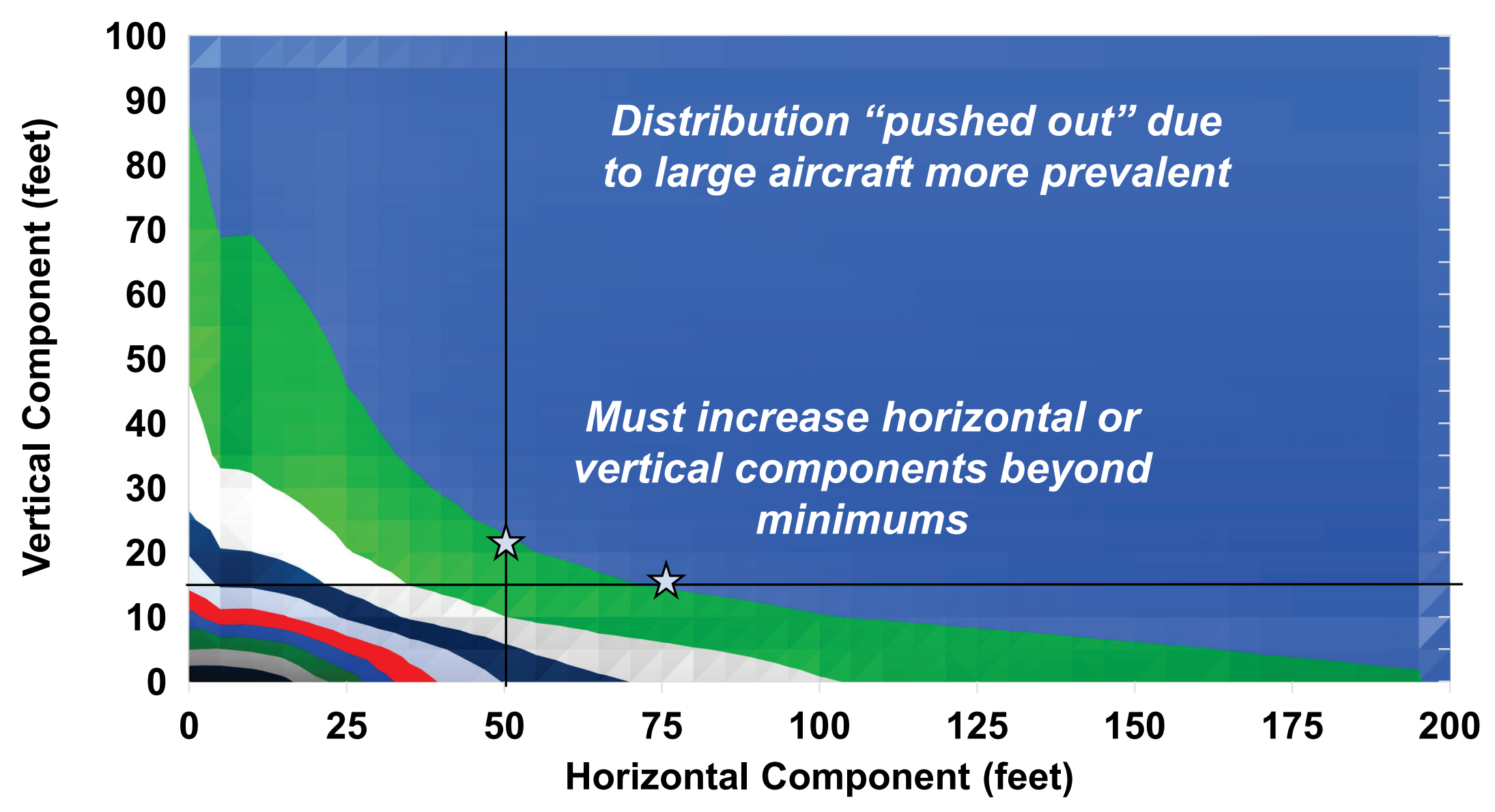

$\backsim 0-0.1 \backsim 0.1-0.2 \quad 0.2-0.3 \square 0.3-0.4 \square 0.4-0.5 \square 0.5-0.6 \square 0.6-0.7 \backsim 0.7-0.8 \backsim 0.8-0.9 \square 0.9-1$ 


\section{sNMAC Preliminary Selections $P(M A C \mid s N M A C) \leq 0.1$}

\section{$P(M A C \mid s N M A C) \leq 0.1$ for uniform and left skew distributions}

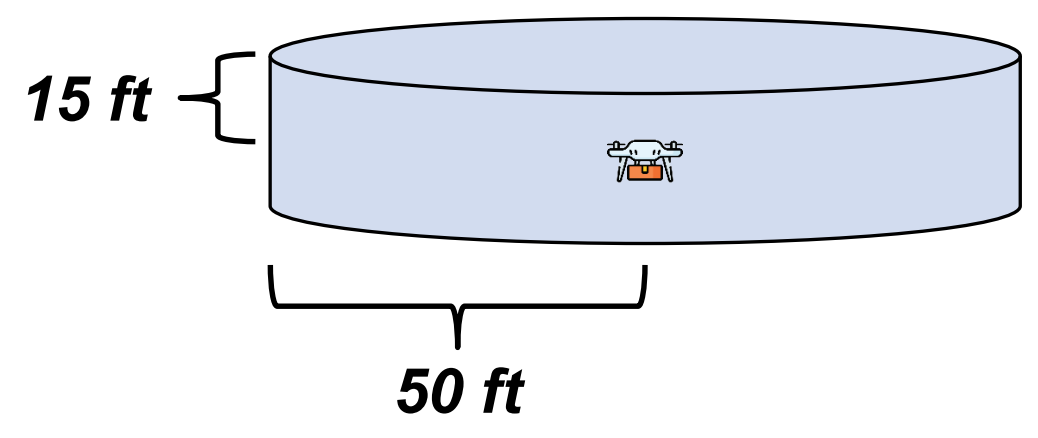

Assume near and mid-term smaller UAS fleet composition best represented by uniform and left skewed width distributions

Right skew width distribution of larger aircraft not sufficiently representative of aircraft fleet but important to assess sensitivity

$P(M A C \mid s N M A C) \leq 0.1$ for all width distributions

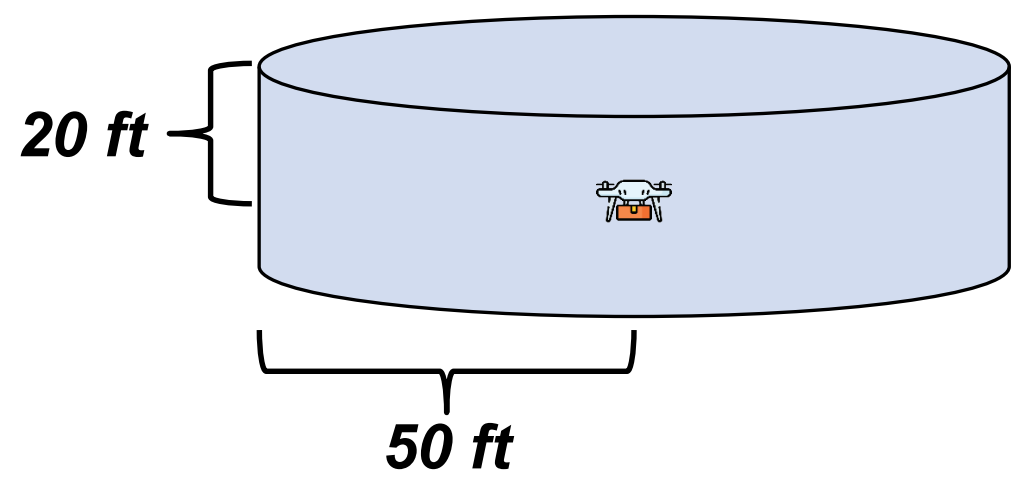

Increase VMD to achieve P(MAC)

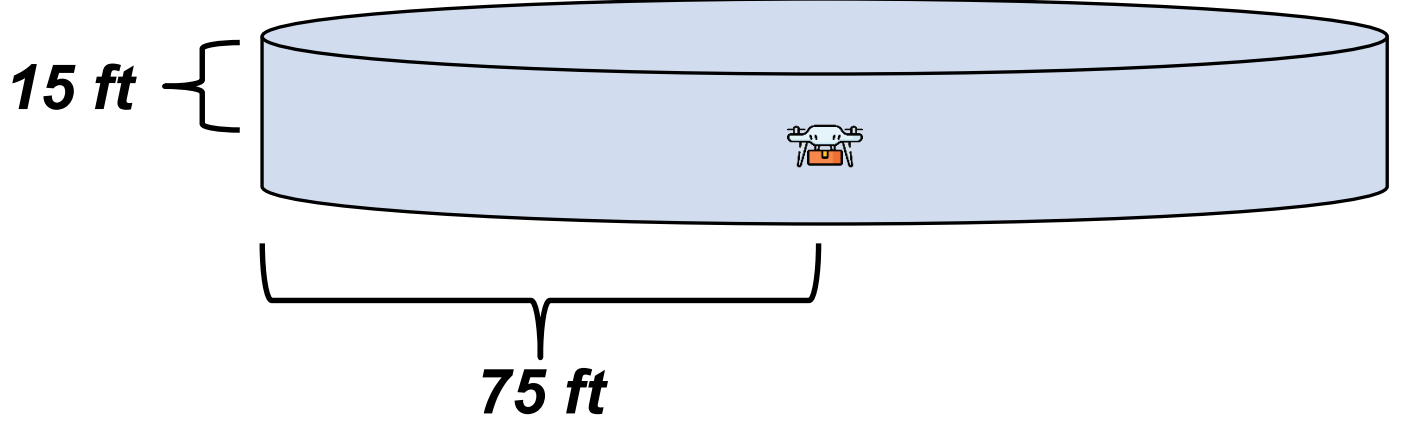

Increase HMD to achieve P(MAC) 
- Overview

- NMAC History

- sNMAC Assumptions

- Method and Results

- Conclusion 


\section{Relationship of NMAC Dimensions to Sensor Noise and Observability}

- Sensor noise makes observation of any event of interest difficult

- For NMACs with manned aircraft, sensor error limits observation of NMACs and makes some NMACs unobserved

- Vertical: Altimeter error

- Horizontal: Ground radar or transponder reply range error

- Two-aircraft altimeter error corresponds to ASARP altimetry error model

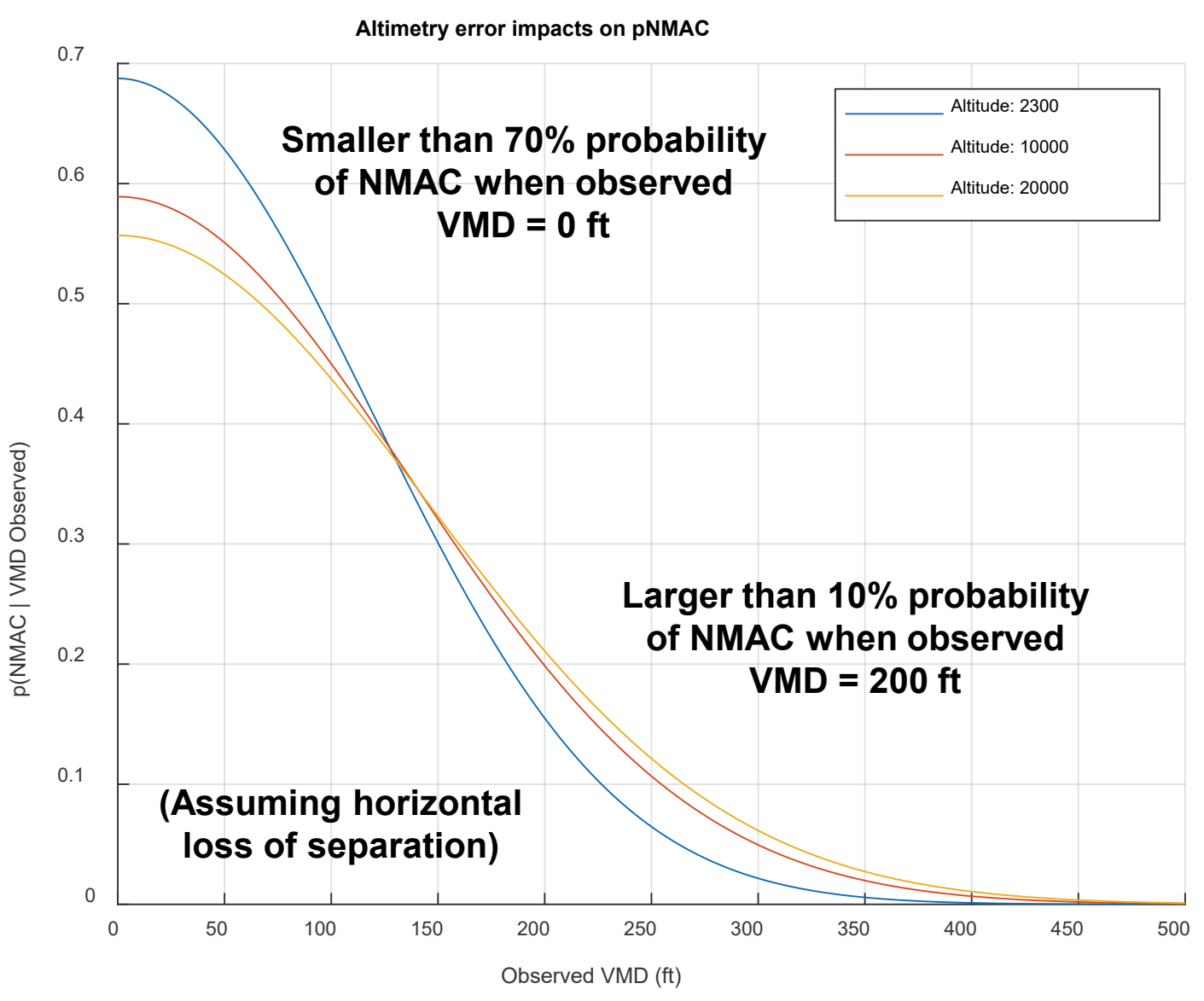




\section{sUAS Altimeter Error Implications sNMAC VMD = 15 feet}

- For preferred sNMAC vertical dimension, sUAS altimeter error has comparable behavior to manned NMAC implications

- sNMAC calculation assumes the same altimeter error for both vehicles

- FAA Remote ID NPRM* proposes only an accuracy of 20 feet for barometric sensors, additional information required to sufficiently assess relationship between position error and SNMAC

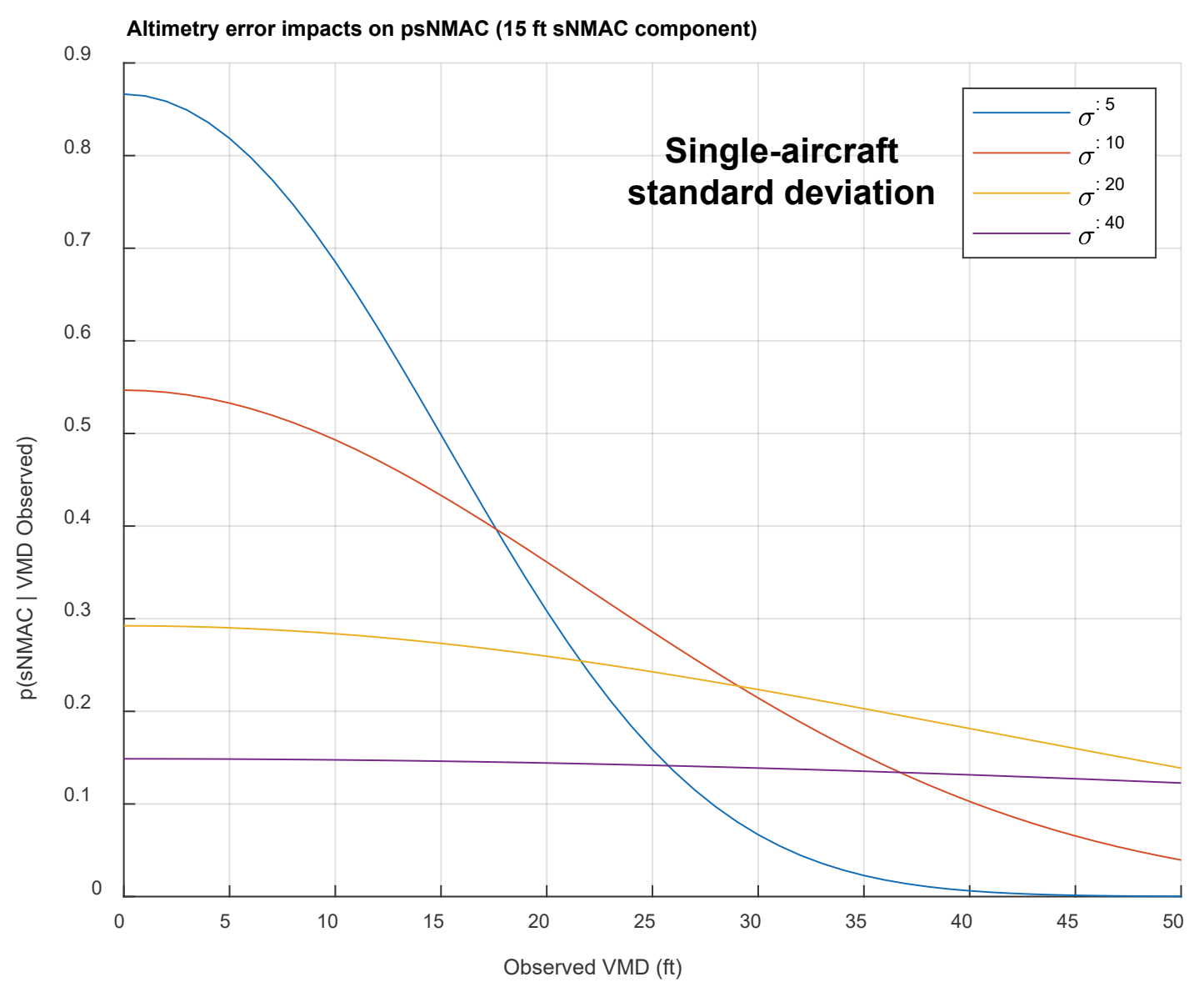

“As proposed in § 89.310(j)(3) for standard remote identification UAS, the reported barometric pressure altitude for the unmanned aircraft and the control station would be required to be accurate to within $\mathbf{2 0}$ feet of the true barometric pressure altitude for pressure altitudes ranging from 0 to 10,000 feet." 


\section{Conclusion}

\section{Community Feedback on sNMAC Requested}

- sNMAC should be a quantitatively derived NMAC analog, informed by stakeholder needs, intended as a metric to assess DAA systems for smaller UAS

- Required to support ACAS sXu DAA development and evaluation

- Preliminary sNMAC dimensions driven by assumed distributions of aircraft size

- Future work needed for harmonization with the FAA and stakeholder needs

- Considerations for the FAA NPRM for Remote ID and effects of position uncertainty

- Simulations with higher fidelity wire-frame models* to better estimate P(MAC)

- Implications for incident reporting were out scope for this preliminary study

- The sNMAC volume, while carefully selected, may change as smaller UAS technology continues to advance and regulations become more developed

- Intent to open source release MATLAB code that generated results to facilitate discussion

- Future work should consider potential policy use of sNMAC 


\section{Thank You!}

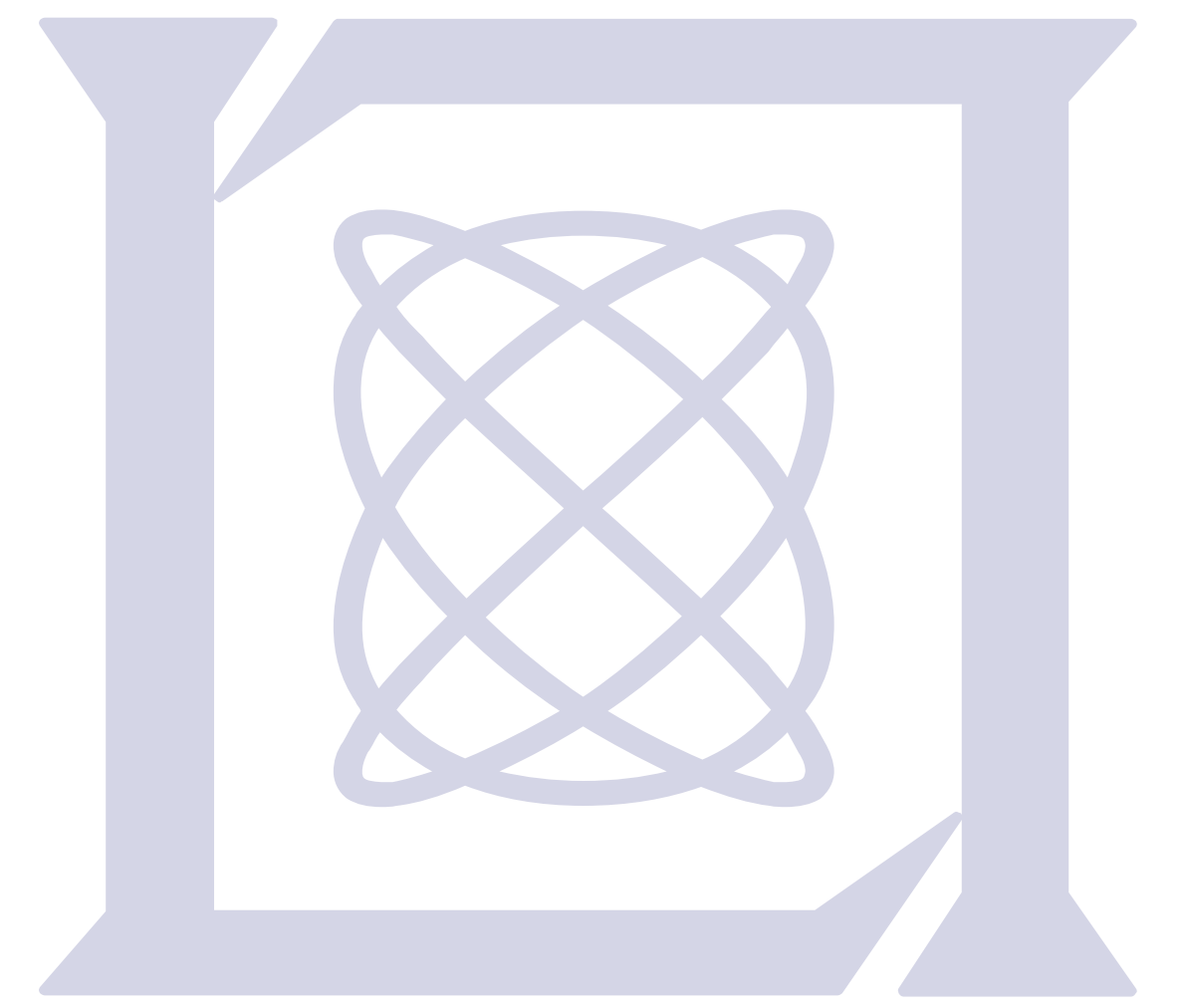

\section{Questions?}

Feedback?

Presenter: Andrew Weinert

Contributors (alphabetical): Luis Alvarez, Michael Owen, Benjamin Zintak

Homeland Protection and Air Traffic Control Division

Email: andrew.weinert@ll.mit.edu 